\title{
Search for sterile neutrinos with the IceCube Neutrino Observatory
}

\author{
The IceCube Collaboration \\ ${ }^{\dagger}$ http://icecube.wisc.edu/collaboration/authors/icrc15_icecube \\ E-mail: marius.wallraffephysik.rwth-aachen.de
}

The IceCube Neutrino Observatory is a $1 \mathrm{~km}^{3}$ Cherenkov detector located at the geographic South Pole. It records several tens of thousands high-energy atmospheric muon neutrino events per year, with energies between about one hundred $\mathrm{GeV}$ and several tens of $\mathrm{TeV}$. This allows a precise measurement of the energy and angular distributions and a search for deviations from the standard expectation that could be due to new physics.

If additional sterile neutrino states exist with mass differences on the order of $1 \mathrm{eV}$ and mix with muon neutrinos, IceCube would observe a deficit compared to the expected neutrino rate in the energy range of a few $\mathrm{TeV}$ due to strong matter effects in the neutrino propagation through Earth. The disappearance depends on the energy and the path of the neutrino through the Earth and thus its zenith angle.

Here, we report on the first exploratory effort to make use of this resonance effect to substantially improve the sensitivity with respect to searches that are based on vacuum oscillations. This paper describes results that are based on a large muon neutrino sample measured by IceCube between 2009 and 2010 in its 59-string configuration.

Corresponding authors: M. Wallraff ${ }^{1 *}$, C. Wiebusch ${ }^{1}$

${ }^{1}$ III. Physikalisches Institut, RWTH Aachen University

The 34th International Cosmic Ray Conference,

30 July- 6 August, 2015

The Hague, The Netherlands

\footnotetext{
* Speaker.
} 


\section{Introduction}

After many decades of research, neutrino flavor oscillations are still an active area of experimental measurements for particle and astroparticle physics. Despite the elusive nature of neutrinos, most parameters that govern the oscillations between the three known neutrino flavors are well known today [1].

Several experimental anomalies, most prominently the $\bar{v}_{\mu} \rightarrow \bar{v}_{\mathrm{e}}$ measurements of the two neutrino beam experiments LSND [2] and MiniBooNE [3], indicate that there could be effects that can not be explained well with the current model of three active neutrinos. One way to explain these measurements is to modify the model by addition of new flavors of neutrinos with a typical mass difference of $\Delta m_{42}^{2} \approx 1 \mathrm{eV}^{2}$. To prevent contradictions to various older measurements such as the well-known limit on the number of light neutrinos from $\mathrm{Z}_{0}$ branching ratio measurements at the LEP [4], these new flavors can not participate in weak interactions and are therefore called sterile [5]. As sterile neutrinos can mix with the active neutrinos, their existence can be tested by the search for disappearance of active neutrinos.

\section{The IceCube Neutrino Observatory}

The IceCube Neutrino Observatory is a neutrino detector located at the Amundsen-Scott South Pole Station. It uses the naturally clear ice of Antarctica as optical medium to observe Cherenkov radiation emitted by charged leptons that have been created by neutrino interactions in the ice. Its active volume of about one cubic kilometer lies at a depth of about 1.5 to $2.5 \mathrm{~km}$ beneath the surface of the ice and is instrumented with 5160 digital optical modules (DOMs) distributed over 86 vertical cables called strings. IceCube was completed in December 2010, but it has already been taking data in the years before with the partially completed detector. The analysis presented here was conducted on data taken with the 59-string configuration between May 2009 and May 2010, called IC-59. This data set consists of approximately 22000 up-going track-like events from chargedcurrent muon neutrino interactions that have good reconstruction quality and energies between about $100 \mathrm{GeV}$ and $50 \mathrm{TeV}$. For details about the event selection, see [6]. A second independent IceCube analysis of comparable sensitivity is close to completion, using the first year of data taken with the final 86-string configuration, and will be reported soon.

\section{Sterile Neutrino Signatures in IceCube}

This analysis is searching for the resonant disappearance of atmospheric muon neutrinos caused by matter-enhanced oscillations during propagation through Earth [7]. For illustrative purposes, the oscillation probability of muon neutrinos to an additional sterile neutrino flavor can be approximated by a two-flavor formalism in the relevant parameter range. The vacuum oscillation probability is then described by

$$
P_{v_{\mu} \rightarrow v_{\mathrm{s}}}=\sin ^{2}\left(2 \theta_{24}\right) \sin ^{2}\left(1.267 \frac{\Delta m_{42}^{2} L}{E_{V}} \frac{\mathrm{GeV}}{\mathrm{eV}^{2} \mathrm{~km}}\right)
$$

where $\theta_{24}$ is the mixing angle between muon neutrinos and sterile neutrinos and $\Delta m_{42}^{2}$ the difference between their squared masses. $E_{v}$ is the neutrino energy and $L$ the baseline, i.e., the distance 


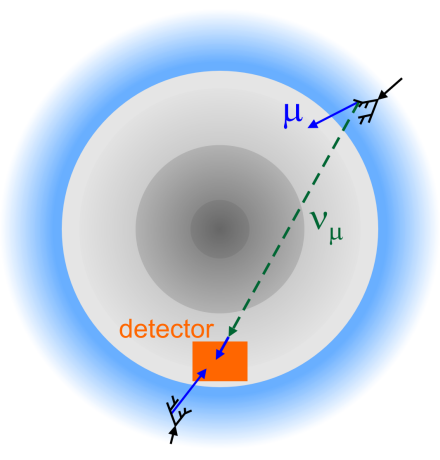

Figure 1: Path of a muon neutrino created in the atmosphere (blue) through Earth, shaded according to density.

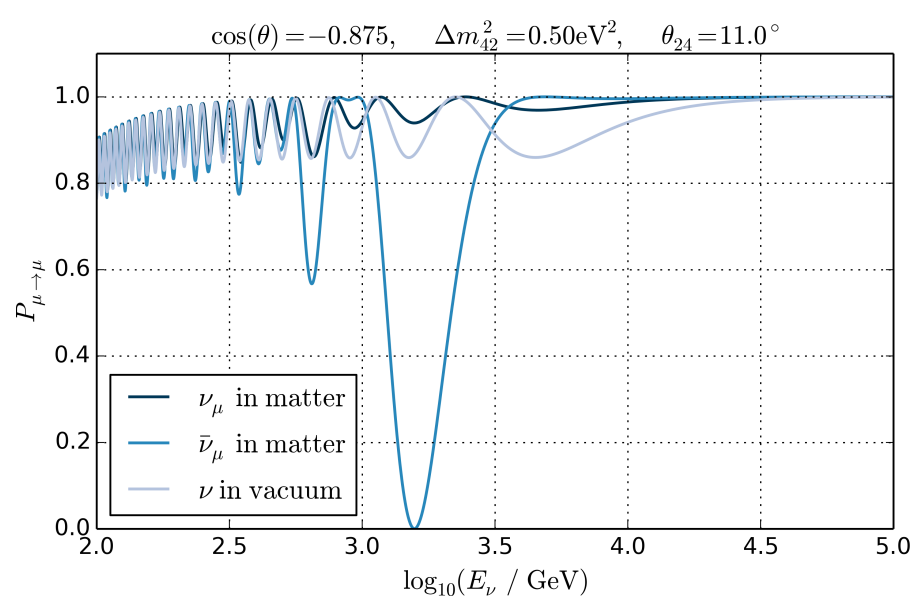

Figure 2: Oscillation probabilities for the neutrino path shown in fig. 1 as a function of its energy in a $3+1$ model.

the neutrino traveled after its creation. The baseline can be calculated from the zenith angle $\theta$ under which the muon travels through the detector (fig. 1). For up-going atmospheric neutrinos, it lies between $500 \mathrm{~km}$ for near-horizontal trajectories and $12800 \mathrm{~km}$, which is the Earth diameter including the atmosphere, for vertical trajectories.

An important difference when compared to conventional atmospheric neutrino analyses is that matter effects inside the Earth are strong. The three conventional flavors can scatter on nucleons and electrons by neutral-current (NC) interactions, and electron neutrinos can additionally scatter elastically on electrons by charged-current (CC) interactions. Sterile neutrinos can not interact weakly by construction. This induces effective mass differences between the flavors that depend on the electron and neutron particle densities. For more detail, see [8]. These induced effective mass differences can cause strong resonances at which the disappearance becomes much stronger than the mixing angle would allow in vacuum. An example is shown in fig. 2. For a new mass state of larger mass than the three known states $\left(0<\Delta m_{32}^{2}<\Delta m_{42}^{2}\right)$ and without $\mathrm{CP}$ violation, the resonance enhances the oscillations in the antineutrino channel and suppresses them for neutrinos. Above $100 \mathrm{GeV}$, the flux of muon neutrinos is larger than that of muon antineutrinos. For this reason, the case described above is the most pessimistic for IceCube and was therefore selected as basis for this initial analysis. It is important to note that the oscillation appears according to the effective mixing as a resonance-like effect, even for small values of vacuum mixing. Therefore, the measurement of atmospheric neutrinos in the relevant energy range has a high sensitivity also to small mixing angles, where vacuum oscillations are small.

The analysis itself does not employ two-flavor approximations, but instead uses full fourflavor propagation based on numeric calculations with the tool nuCraft [9]. The rotation matrix with $\theta_{24}$ has been multiplied to the left side of the $4 \times 4$ PMNS matrix. All other sterile mixing angles have been set to zero; $\theta_{14}$ controls the mixing between electron and sterile neutrinos and is negligible for the atmospheric muon neutrino disappearance as seen by IceCube. For non-zero $\theta_{24}$, the tau-sterile angle $\theta_{34}$ influences oscillation amplitude and shape roughly similar to $\theta_{24}$ [10]. It also results in a substantial fraction of the muon neutrinos oscillating to tau neutrinos instead of sterile neutrinos. However, most tau neutrinos cause cascade-like signatures and the 

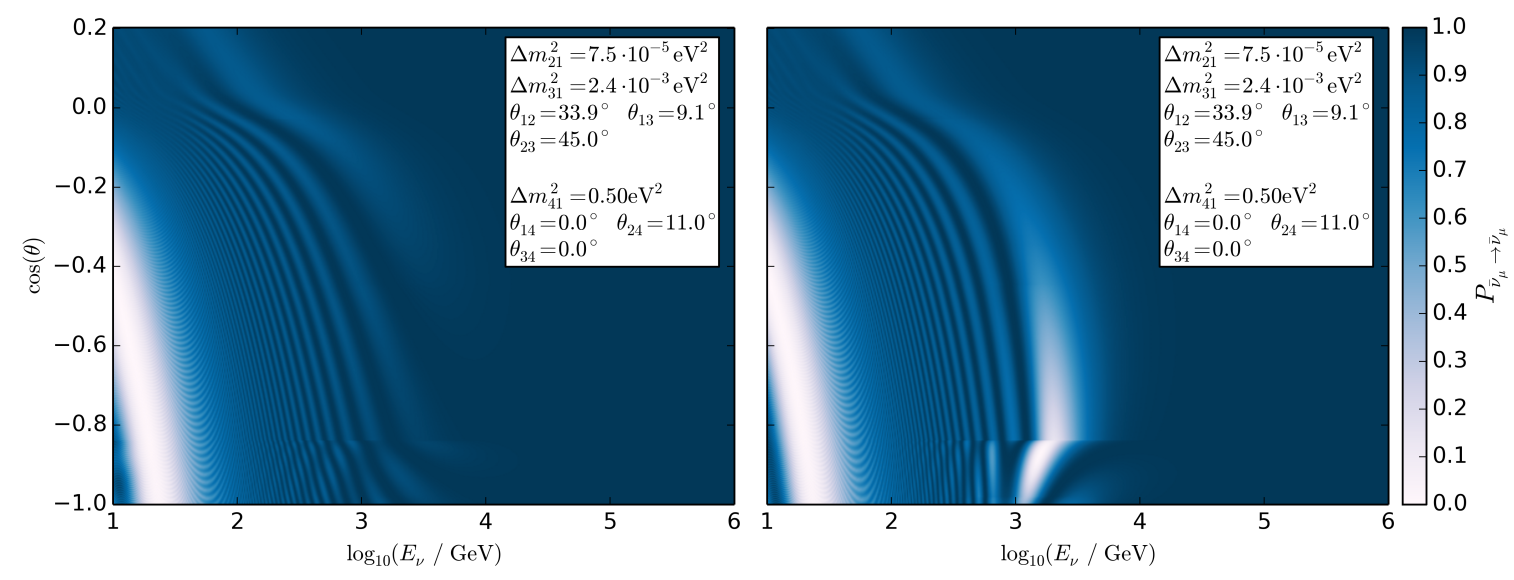

Figure 3: Probability for atmospheric muon neutrinos (left) and muon antineutrinos (right) for the particle to reach the detector in the same state, with one sterile state of neutrinos, parameters as specified in the plot.

remaining $15 \%$ produce only faint muon tracks, so they are strongly suppressed by this event selection, which favors track-like signatures. For these reasons, $\theta_{34} \neq 0$ leads to signatures that are sufficiently similar to (and stronger than) those with $\theta_{34}=0$ to warrant this parameter to be omitted for now. Also, models with more than one sterile flavor have not been included into this analysis to keep it computationally feasible. The signatures of models with multiple sterile flavors can be approximated well by superpositions of $3+1$ signatures and are therefore much more similar to $3+1$ models than to $3+0$ models, so they would still be observable by this $3+1$ analysis [10].

An example for an atmospheric muon neutrino disappearance signature investigated by this analysis is shown in fig. 3. The parameters of the sterile neutrino have been chosen to be $\Delta m_{42}^{2}=$ $0.5 \mathrm{eV}^{2}, \theta_{24}=11^{\circ}$, because these values have not yet been excluded by other experiments, but are large enough to serve for illustrative purposes. In the antineutrino channel, a strong, resonant oscillation minimum can be seen at about $4 \mathrm{TeV}$ for the particles that traversed the inner core of the Earth. The signature shifts to higher (lower) neutrino energies for larger (smaller) $\Delta m_{42}^{2}$, and it becomes stronger and shifts closer to the horizon for larger $\theta_{24}$.

\section{Analysis Method}

The aim of the analysis is to determine or to constrain $\theta_{24}$ and $\Delta m_{42}^{2}$. This is accomplished by a forward-folding method where the measured muon neutrino rate as a function of reconstructed energy and direction is compared with expectations from simulation. We use a two-dimensional likelihood ratio test, binned in the reconstructed values for $\cos (\theta)$ and $\log _{10}\left(E_{\mu}\right)$ :

$$
\Lambda=\frac{\sup _{\widehat{\xi}} \mathscr{L}\left(\theta_{24}, \Delta m_{42}^{2}, \widehat{\xi}\right)}{\sup _{\widehat{\theta_{24}}, \widehat{\Delta m_{42}^{2}}, \widehat{\xi}} \mathscr{L}\left(\widehat{\theta_{24}}, \widehat{\Delta m_{42}^{2}}, \widehat{\xi}\right)} .
$$

Here, $\mathscr{L}$ denotes the likelihood, which is a standard Poissonian likelihood function of the event numbers in all bins, and sup indicates the maximized likelihood. The results were compared and verified with a likelihood formulation that also takes into account statistical uncertainties of the 
simulated reference histograms [13]. $\xi$ denotes an ensemble of nuisance parameters that are optimized independently at each point in the physics parameter space for best agreement with the experimental data [14].

For energies that are relevant for this analysis, the zenith angle $\theta$ can be measured with good accuracy, to better than one degree. The neutrino energy $E_{v}$ can be estimated by measuring the energy loss of its induced muon. The energy resolution is limited as the muon does not inherit the full energy of the neutrino, and the interaction might have happened far outside the detector such that the muon already lost a significant part of its energy. However, as this analysis employs forward folding, it does not rely on the individual values of the reconstructed neutrinos as long as the reconstruction algorithm behaves identical for the ensembles of experimental and simulated data. The data set is binned in two dimensions, with 25 bins in $\cos (\theta)$ and 13 bins in $\log _{10}\left(E_{\mu}\right)$.

The effects of systematic uncertainties on the expectation values of event rates in each zenithenergy bin have been parametrized and included into the likelihood function as continuous parametrizations of the corresponding nuisance parameters $\xi$. In contrast to the two physics parameters $\theta_{24}$ and $\Delta m_{42}^{2}$, the fit results for nuisance parameters can not be considered to be measurements of the physical quantities as they are free parameters in the numerator and denominator of the likelihood ratio for every point of the likelihood scan. Furthermore, they can be highly degenerate in their effect on the description of the data. Their purpose is to account for all systematic uncertainties to avoid a bias in the measurement of the physics parameters.

The likelihood maximization is performed by a scan in the parameter range of $\Delta m_{42}^{2}=10^{-2.0}$ $\ldots 10^{0.7} \mathrm{eV}^{2}$ and $\theta_{24}=0 \ldots 42.5^{\circ}$, and the differences between two times the logarithmic likelihood (LLH) values $\Lambda$ from (4.1) for a given pair of parameters and the point of best fit are then used as a test statistic. According to Wilks' theorem, this follows a $\chi^{2}$ distribution with two degrees of freedom if the minimum of the LLH landscape can clearly be associated to a specific pair of parameters [15]. However, this precondition of Wilks' theorem is not strictly fulfilled for the null hypothesis, i.e., for the case that there are no sterile neutrinos mixing with muon neutrinos, and we have determined the small deviations from the predicted $\chi^{2}$ distribution by ensemble tests. For the construction of confidence regions we use the more conservative test statistics from these ensemble tests.

\section{Systematic Uncertainties}

For this challenging measurement that relies on the agreement between simulated and measured distribution of energy and zenith, a correct handling of systematic uncertainties is crucial. Because of the large possible parameter space of sterile neutrinos, it is not possible to define an off-region that is known to be free of possible signal influence and could be used for the determination of nuisance parameters. At this point, most, but not yet all, relevant uncertainties are included, so the results are still preliminary. The nuisance parameters that are included are the total rate normalization, the cosmic-ray spectral index that affects the slope of the energy distribution, the kaon-pion ratio in cosmic-ray interactions that affects the angular distribution, the antineutrinoneutrino ratio that affects angular distribution and strength of the oscillation effect, and the relative optical efficiency of the light detection that affects the energy scale. All of them are implemented with a weak Gaussian prior, larger than or equal to the estimated uncertainty, to be particularly con- 

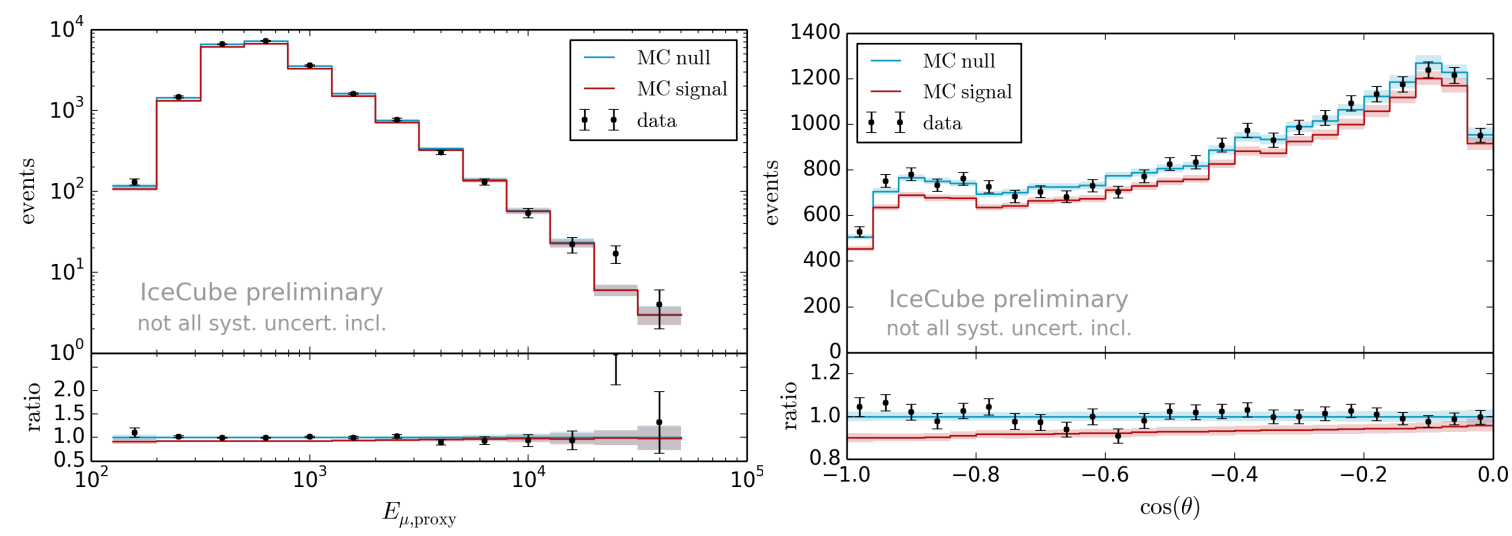

Figure 4: Distributions of reconstructed energy and reconstructed zenith angle, each marginalized over the other variable, respectively; the error band indicates the statistical uncertainty on the Monte-Carlo prediction. Shown are measured experimental data, as well as Monte-Carlo predictions for the null hypothesis and for our benchmark signal model from the previous figures. Note that for demonstration purposes the nuisance parameters have not been fitted for the benchmark model.

\begin{tabular}{lrr} 
uncertainty & default value & prior \\
\hline total rate normalization & 1.00 & 0.40 \\
cosmic-ray spectral index & 2.65 & 0.05 \\
cosmic-ray kaon-pion ratio & 1.00 & 0.10 \\
antineutrino-neutrino ratio & 0.440 & 0.022 \\
relative optical efficiency & 1.00 & -
\end{tabular}

Table 1: Nuisance parameters and their priors

servative in this first analysis and only to improve the numerical convergence of the fit; see table 1. The atmospheric neutrino flux is based on a parametrization of the model by [16], extending it to higher energy. This flux is modified by changes of the above nuisance parameters. In addition, small changes in the zenith distribution related to atmospheric temperature variations have been estimated based on satellite data and corrected for. Currently not included is a variation of the ice model parameters that describe the propagation of photons. This propagation is modeled according to [17] and a comparison with [18] is underway. Uncertainties on the Earth's matter density and neutrino interaction cross-sections are negligible compared to other uncertainties and have not been included.

\section{Results}

Figure 4 shows the distribution of experimental data as marginalizations of the reconstructed zenith angle and energy distributions in comparison with the simulation of the null hypothesis with fitted nuisance parameters. The experimental result is well described with the simulation, excluding a strong disappearance signal. For comparison, the figure also includes predictions for the benchmark model described in section 3, with the same nuisance parameter values as fitted for the null hypothesis. The signature of this model is clearly distinguishable from the null hypothesis. 


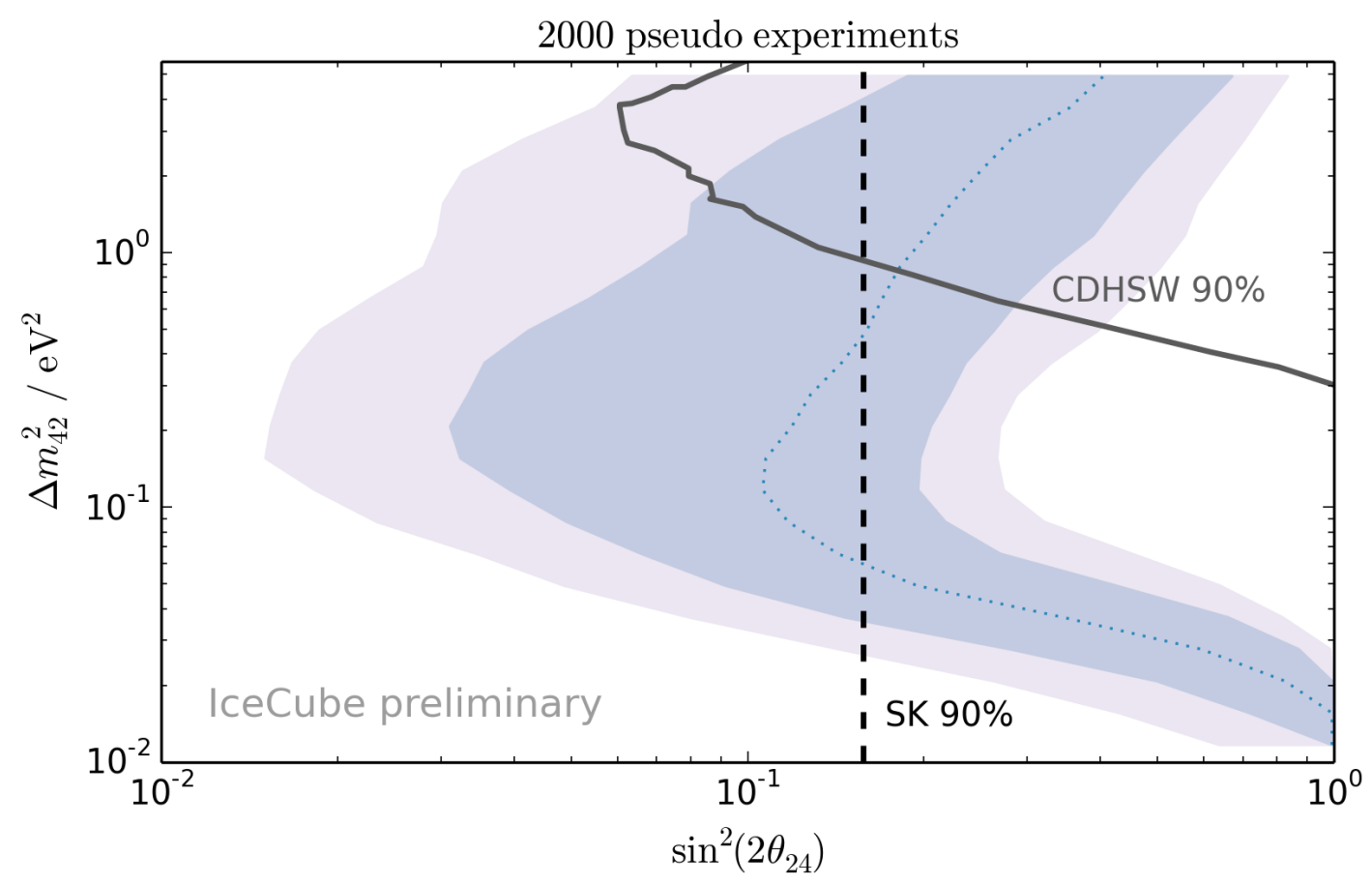

Figure 5: Expected region of 90\% C.L. exclusion sensitivity of this analysis for the null hypothesis in comparison to the exclusion by other experiments [5][12]. For the Super-K contour, $\theta_{14}=0$ was assumed. The contours are indicating the median (dotted curve), the $68 \%$ (darker blue) and $90 \%$ (lighter blue) ranges for the $90 \%$ C.L. exclusions from an ensemble of 2000 pseudo-experiments. Not all systematic uncertainties have been fully included yet.

When the nuisance parameters get fitted, the distributions become more similar, but the tension of the nuisance parameters with their expected values allows us to retain much of our sensitivity.

As discussed in section 3, this analysis is, unlike those of other experiments, primarily sensitive to the matter resonance effect. As a result, we expect fluctuations in the sensitivity for the vacuum oscillation parameters. In order to quantify these fluctuations we have analyzed a series of pseudo-experiments based on the simulated null hypothesis. Figure 5 shows this expected range of exclusions. In a $\Delta m_{42}^{2}$ range between 0.1 and $1 \mathrm{eV}^{2}$, the analysis can significantly extend existing limits to values of $\sin ^{2}\left(2 \theta_{24}\right)=0.1$ or even below. Experimental results will be reported soon.

\section{Conclusion and Outlook}

With the large statistics of well-reconstructed tracks induced from atmospheric muon neutrinos that IceCube measures each year, it is possible to search for disappearance effects caused by sterile neutrinos that mix with muon neutrinos. The energy- and zenith-dependent signature of these neutrino oscillations gets strongly enhanced by matter effects inside the Earth and increases IceCube's sensitivity in the mass-splitting range of about $\Delta m_{42}^{2}=0.01 \ldots 1.0 \mathrm{eV}^{2}$.

Here, the sensitivity of a first IceCube analysis are presented with data from IceCube in its 59string configuration. The expected exclusion limits significantly constrain the allowed parameter space for sterile neutrinos and substantially improve previous limits. In the near future, an anal- 
ysis based on IceCube data taken over multiple years with a selection optimized for the relevant energy range could further improve these limits significantly. The high sensitivity of this analysis demonstrates the good performance of IceCube and its capability to address fundamental questions of particle physics.

\section{Acknowledgments}

In addition to the general IceCube acknowledgments in the special section of these proceedings, we would like to thank the IT Center of the RWTH Aachen University for their grant of computing resources.

\section{References}

[1] J. Beringer et al., Phys. Rev. D 86 (2012) 177-198 doi:10.1103/PhysRevD.86.010001.

[2] A. Aguilar et al., Phys. Rev. D 64 (2001) 112007 doi:10.1103/PhysRevD.64.112007.

[3] A.A. Aguilar-Arevalo et al., Phys. Rev. Lett. 110 (2013) 161801 doi:10.1103/PhysRevLett.110.161801.

[4] R.W.L. Jones et al., Phys. Rep. 427 5-6 (2006) 257-454 doi:10.1016/j.physrep.2005.12.006.

[5] K.N. Abazajian et al., Light Sterile Neutrinos: A White Paper, hep-ph (2012) [arXiv:1204.5379].

[6] M.G. Aartsen et al., Phys. Rev. D 89062007 (2014) doi:10.1103/PhysRevD.89.062007, [arXiv:1311.7048].

[7] H. Nunokawa, O.L.G. Peres and R. Zukanovich Funchal, Phys. Lett. B 562279 (2003) [arXiv:hep-ph/0302039].

[8] T.K. Kuo and J. Pantaleone, Rev. Mod. Phys. 61 (1989) 937-977 doi:10.1103/RevModPhys.61.937.

[9] M. Wallraff and C. Wiebusch, Calculation of oscillation probabilities of atmospheric neutrinos using nuCraft, Comput. Phys. Commun. (forthcoming) [arXiv:1409.1387].

[10] D. Hellwig and M. Wallraff, Calculation of oscillation probabilities of atmospheric neutrinos with additional sterile mass states using nuCraft, IceCube Internal Report (2013) icecube/201303001.

[11] A. Esmaili, F. Halzen and O.L.G. Peres, Exploring $v_{\tau}-v_{s}$ mixing with cascade events in DeepCore, JCAP 1307048 (2013) [arXiv:1303.3294].

[12] K. Abe et al., Phys. Rev. D 91052019 (2015) doi:10.1103/PhysRevD.91.052019.

[13] D. Chirkin, Likelihood description for comparing data with simulation of limited statistics, Instrumentation and Methods for Astrophysics (2013) [arXiv:1304.0735].

[14] G. Cowan et al., EPJ C 71 (2011) 1554 doi:10.1140/epjc/s10052-011-1554-0.

[15] S.S. Wilks, Ann. Math. Statist. 9 (1938) 60-62 doi:10.1214/aoms/1177732360.

[16] M. Honda, T. Kajita, K. Kasahara, S. Midorikawa and T. Sanuki, Phys. Rev. D 75, 043006 (2007) [arXiv:astro-ph/0611418].

[17] M.G. Aartsen et al., Nucl. Instrum. Meth. A 711 (2013) 73 [arXiv:1301.5361].

[18] M.G. Aartsen et al., Evidence of optical anisotropy of the South Pole ice, 33nd ICRC (2013) [arXiv:1309.7010]. 